\title{
RADIOISOTOPE-POWERED IMPULSE RADIO FREQUENCY SENSOR NODE
}

\author{
S. Tin, R. Duggirala, A. Lal, and C. Pollock
}

SonicMEMS Laboratory, School of Electrical and Computer Engineering, Cornell University

Ithaca, NY, USA

\begin{abstract}
We demonstrate a radioactive thin-film powered wireless sensor node which can transmit an RF signal coded with the environmental information gathered by a passive capacitive sensor. Sensor nodes operate for several decades and transmit data over RF links at powers of 100-1000 milliWatts. In our active sensor node, radioisotope energy emitted in electrons from ${ }^{63} \mathrm{Ni}$ thin films is used to electrostatically charge a cantilever over several minutes, and the stored electrical energy is released over a nano-second time scale, achieving a collected-to-released power amplification of $10^{9}$ (10 mins/10ns). Even when the radioisotope emitted power is nanowatts from a benign amount of ${ }^{63} \mathrm{Ni}$, over $500 \mathrm{~mW}$ of transmitted RF power has been remotely detected. Since the half life of ${ }^{63} \mathrm{Ni}$ is 100 years, this RF transmitter can work autonomously for several decades. In our prototype wireless sensor node, a humidity sensitive polymer capacitance is used to demonstrate sensor node functionality. A transmitted frequency shift of $4.27 \mathrm{MHz}$ over $150 \mathrm{MHz}$ is achieved when the humidity changes from $22 \%$ to $97 \%$.
\end{abstract}

\section{INTRODUCTION}

A critical requirement for the success of autonomous remote systems is the realization of miniature power sources with long lifetimes, especially for sensor networks working in harsh, inaccessible environments. While traditional power sources can only work up to several years without replacement or refueling, radioactive isotope power sources can work for 100 years with their performance mostly unaffected by environment. This is possible due to the high energy density $\left(\sim 10^{5} \mathrm{~kJ} / \mathrm{m}^{3}\right)$ and long halflife (100.2 years) of the ${ }^{63} \mathrm{Ni}$ radioisotope fuel used in the device. Nickel-63 emits $\beta$-particles with an average energy $E_{\text {avg }}=17.3 \mathrm{keV}$ and a maximum penetration depth $<10 \mu \mathrm{m}$ in solids. As a result, devices powered by ${ }^{63} \mathrm{Ni}$ thin-films can be deployed safely with microscale shielding.

The power requirements for a typical low-power wireless sensor node are shown in Figure 1. Power requirements are 1-10 $\mathrm{nW}$ for retaining memory state, $0.1-1 \mathrm{~mW}$ for periodic sensing and processing, and 1-100 $\mathrm{mW}$ power for periodic wireless

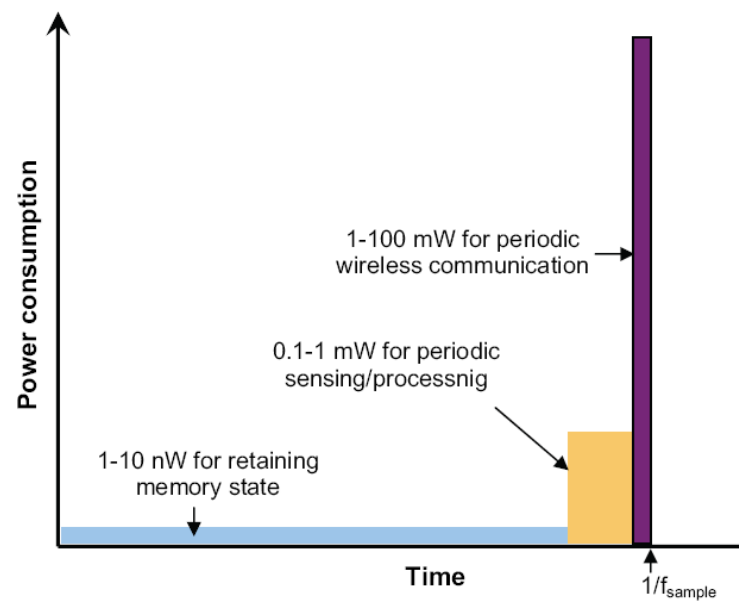

Figure 1: Schematic illustrating the power requirements of typical periodically sampling low power wireless sensor microsystems [1]. communication. The power to retain memory state can be provide by betavoltaics as reported in [2] even with the low activity radioactive thin films presented here. Furthermore, MEMS radioisotope-powered piezoelectric power generator [3] can generate output power needed for periodic sensing and processing. In this paper, we focus on the radioactive RF power generation that can be used as a CMOS compatible wireless beacon and communications. A sensing network can thus be powered entirely with thin films of low activity radioisotope.

Nickel-63 radioisotope actuated reciprocating piezoelectric [3] and piezoelectric unimorph cantilevers [4] have been previously reported to generate RF pulses upon radioisotope discharge. These were limited to pulses generated from dielectric waveguides integral to the self-reciprocating cantilever. In this paper, the cantilever is a simple micromachined gold cantilever limiting its function only for charge collection and release (Figure 2). ${ }^{63} \mathrm{Ni}$ isotope source have been used to bias sensors [5] and generate RF signal [6]. In this paper we demonstrate a fully selfpowered sensor node with hundreds of milliwatts of RF transmitter power output. An RF equivalent circuit model of the selfreciprocating cantilever system has been developed that agrees with the experimentally observed frequency output versus sensor capacitance.

\section{PRINCIPLE OF OPERATION}

As illustrated in Figure 2, a gold cantilever is placed about $500 \mu \mathrm{m}$ above a ${ }^{63} \mathrm{Ni}$ radioactive thin film with $1.5 \mathrm{mCi}$ activity. Positive charges are accumulated on an electrically isolated ${ }^{63} \mathrm{Ni}$ thin film due to the continuous emission of $\beta$-particles (electrons), which are collected on the gold cantilever. The accumulated charge increases with time, increasing the electrostatic force which eventually pulls the cantilever into the radioisotope thin-film. When the gap between the cantilever and radioactive source is small enough, arc discharge through the gap occurs. As the electrostatic force is eliminated, the beam oscillates, and the process repeats itself.

When the gap between the cantilever and radioactive source is $500 \mu \mathrm{m}$, the pull-down cycle requires 3 minutes. As the cantilever approaches the source, tunneling and direct conduction-based current results in a pulse that is very short in time $(<1 \mathrm{~ns})$. The energy integrated over the reciprocation period is released in a very

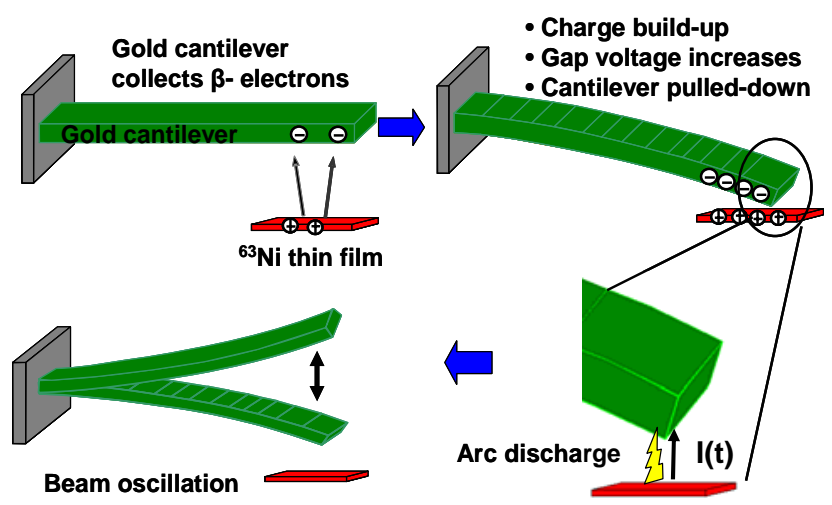

Figure 2: The beta-radioisotope actuated reciprocation of gold cantilever 
(a)

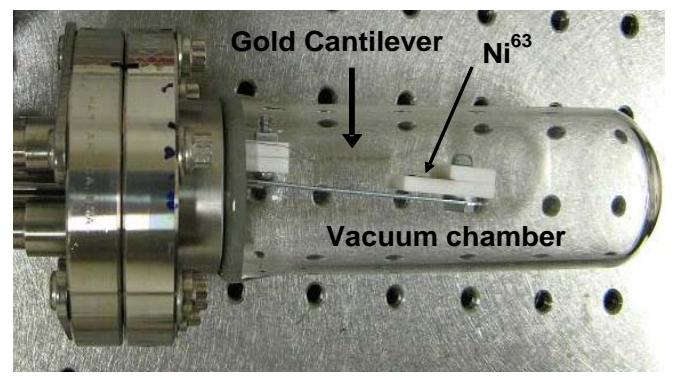

(b)

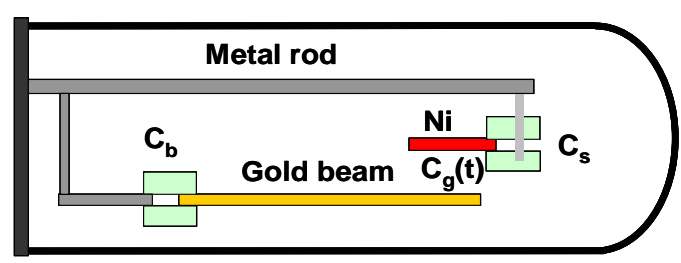

(c)

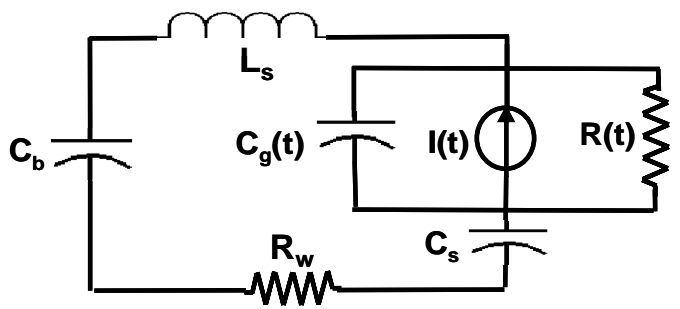

(d)

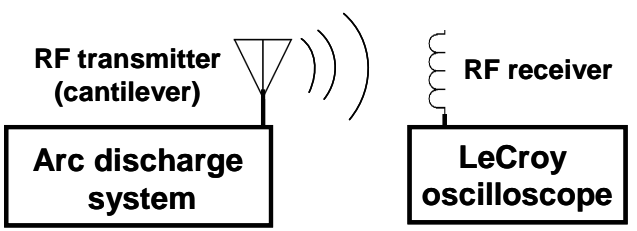

Figure 3. (a) Photograph, (b) schematic, and (c) equivalent LC circuit of the self-powered wireless discharge system; (d) schematic of wireless detection system

short time allowing us to greatly amplify the power from the radioactive source. The discharge current pulse passes through the $\mathrm{RF}$ circuit formed by the device exciting its impulse response.

As shown in Figure 3(a), a gold cantilever $(5 \mathrm{~cm} \times 0.8 \mathrm{~cm} \times 300 \mu \mathrm{m})$ and ${ }^{63} \mathrm{Ni}$ source discharging system is

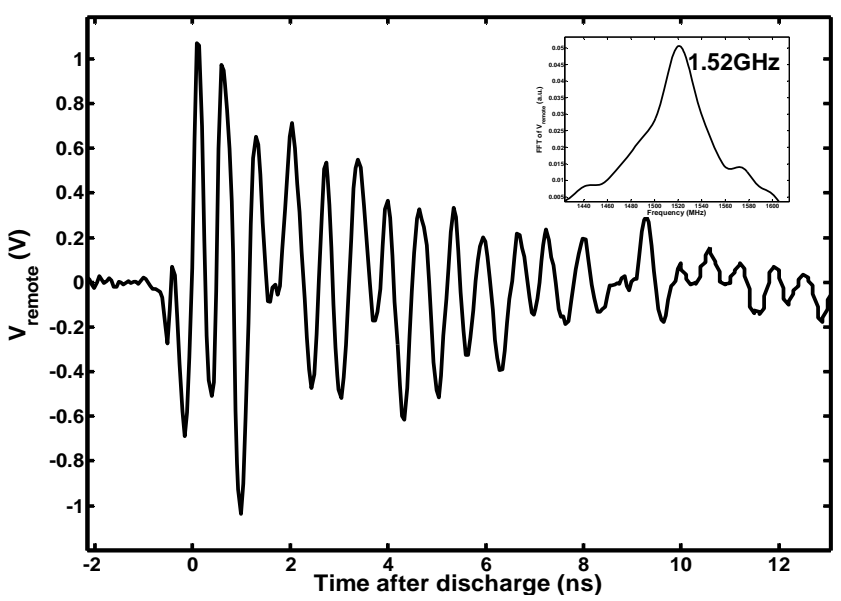

Figure 4. Magnitude and frequency of the remotely detected $R F$ signal of the arc discharge system
Table 1: Representation and measured values of $R, L, C$ components in circuit model in Figure 3(c)

\begin{tabular}{|c|c|c|}
\hline Component & Representation & Value \\
\hline $\mathrm{C}_{\mathrm{s}}$ & Source holder capacitance & $8.3 \mathrm{pF}$ \\
\hline $\mathrm{C}_{\mathrm{b}}$ & Beam holder capacitance & $8.3 \mathrm{pF}$ \\
\hline $\mathrm{R}_{\mathrm{w}}$ & $\begin{array}{c}\text { Resistance of metal beams } \\
\text { and rod }\end{array}$ & $0.1 \mathrm{ohm}$ \\
\hline $\mathrm{L}_{\mathrm{s}}$ & $\begin{array}{c}\text { Equivalent inductance of } \\
\text { the arc discharge system }\end{array}$ & $2.4 \mathrm{nH}$ \\
\hline $\mathrm{C}_{\mathrm{g}}(\mathrm{t})$ & Air gap capacitance & $\begin{array}{c}\text { Time } \\
\text { dependent }\end{array}$ \\
\hline $\mathrm{I}(\mathrm{t})$ & Air gap current & $\begin{array}{c}\text { Discharge } \\
\text { pulse }\end{array}$ \\
\hline $\mathrm{R}(\mathrm{t})$ & $\begin{array}{c}\text { Equivalent resistance across } \\
\text { the air gap }\end{array}$ & $\begin{array}{c}\text { Arc } \\
\text { resistance }\end{array}$ \\
\hline
\end{tabular}

housed inside a small glass vacuum chamber that is evacuated and sealed. Connected to a vacuum pump, the chamber is pumped down to $10^{-3}$ mTorr. Both the gold cantilever and the ${ }^{63} \mathrm{Ni}$ source are held in place with quarter-inch thick Teflon plates, which forms a capacitor $C_{b}$ at the beam holder, and a capacitor $C_{s}$ at the radioactive source holder (Figure $3(\mathrm{~b})$ ). $\mathrm{C}_{\mathrm{g}}(\mathrm{t})$ is the air-gap capacitance at any given time t. An RF receiver (air-core coils with 21 turns and $1.5 \mathrm{~cm}$ inner diameter) is connected to a high bandwidth oscilloscope (LeCroy WaveMast 8500) placed outside the chamber to detect and record the RF signal (Figure 3(d)). RF signal with frequency of $1.52 \mathrm{GHz}$, which lasts for around $10 \mathrm{~ns}$, is remotely detected upon cantilever discharge (Figure 4.)

An LC circuit model is developed for the wireless RF discharge system (Figure 3(c)) with the measured values of the components list in Table 1. When an arc discharge occurs across the air gap, a sub-nanosecond high power current pulse is released which generates oscillations in the RLC circuit at hundreds of megahertz for hundreds of nanoseconds. After the discharge, the cantilever is effectively at rest because of mechanical inertia. Then the gap between the cantilever and the source is very small, which means the value of $\mathrm{C}_{\mathrm{g}}$ is very large at that moment compared to other capacitors in the circuit.

To verify the circuit model, the circuit in Figure 3(c) is simulated in SPICE with the capacitance across the discharge air

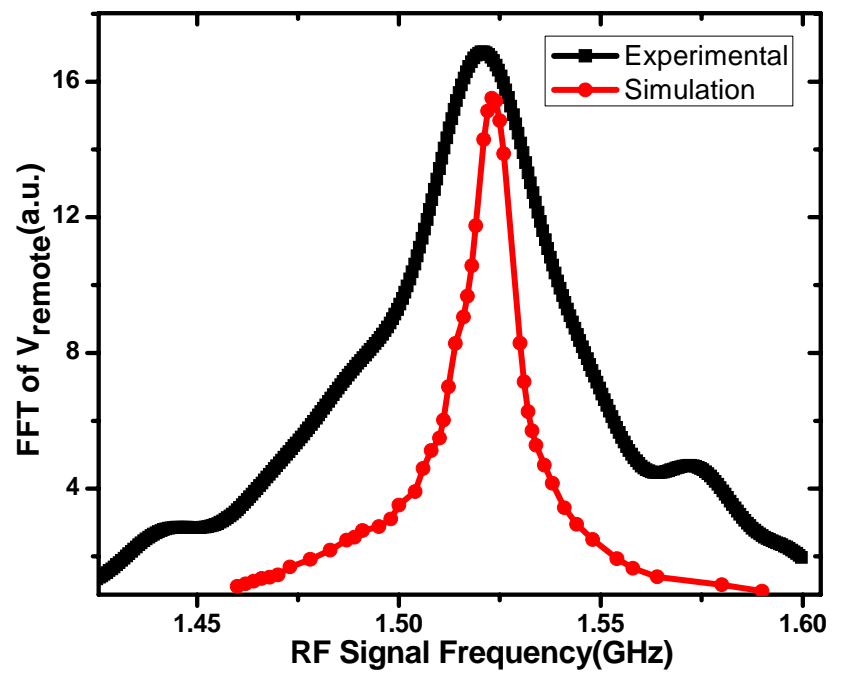

Figure 5. Resonance response of the arc discharge system from SPICE simulation and experimental measurement. 
gap, $\mathrm{C}_{\mathrm{g}}$ is estimated to be $100 \mathrm{pF}$ with contact area of $100 \mu \mathrm{m}^{2}$. Capacitance $\mathrm{C}_{\mathrm{g}}$ " $\mathrm{C}_{\mathrm{s}}$ and acts as a short compared to other impedances in the circuit. $\mathrm{R}(\mathrm{t})$ is relatively large and is ignored in this simulation, as it is shorted out by $\mathrm{C}_{\mathrm{g}}$ at the time scales involved. The values of other capacitors and inductors used in the simulation are listed in Table 1 . The cantilever reciprocation period is 3 minutes with $0.64 \mathrm{~cm}$ of the cantilever area exposed to the radiation. Therefore, with $9 \mathrm{pA}(1.5 \mathrm{mCi})$ electron current, the totally charge across the air gap at the time of arc discharge is $1 \mathrm{nC}$. Assuming the discharge lasts 1ns, the average discharge current will be $1 \mathrm{~A}$. A pulse current of $1 \mathrm{~A}$ with rising and falling time of 0.1 ns (time estimated from experimental values) and duration time of 1 ns are chosen to excite the LC circuit in the simulation (resonance frequency of the circuit is independence of pulse magnitude and duration). The simulated resonance frequency of the LC circuit is plotted in Figure 5, together with the frequency of remotely measured RF signal from device shown in 3(a). The simulation result agrees with the experimental result, both of which are around $1.52 \mathrm{GHz}$.

\section{EXPERIMENTAL SETUPS AND RESULTS}

The high power pulses from the arc discharge system can be utilized to generate wireless RF signals detectable over long distances. As shown in Figure 1, wireless communication is the most power consuming process in the sensor network. Equipped with a self-powered RF signal source, one needs to modulate the RF output with the sensing signal.

In the system shown in Figure 3(a), all the parameters inside the glass chamber have fixed values. The RF frequency can only be modulated with components outside the chamber. As shown in Figure 6(a), external capacitor is connected to the arc discharge system. In the equivalent circuit (Figure 6(b)), the circuit inside the dotted box is the arc discharge system, while $\mathrm{C}_{\mathrm{SC}}$ is the external

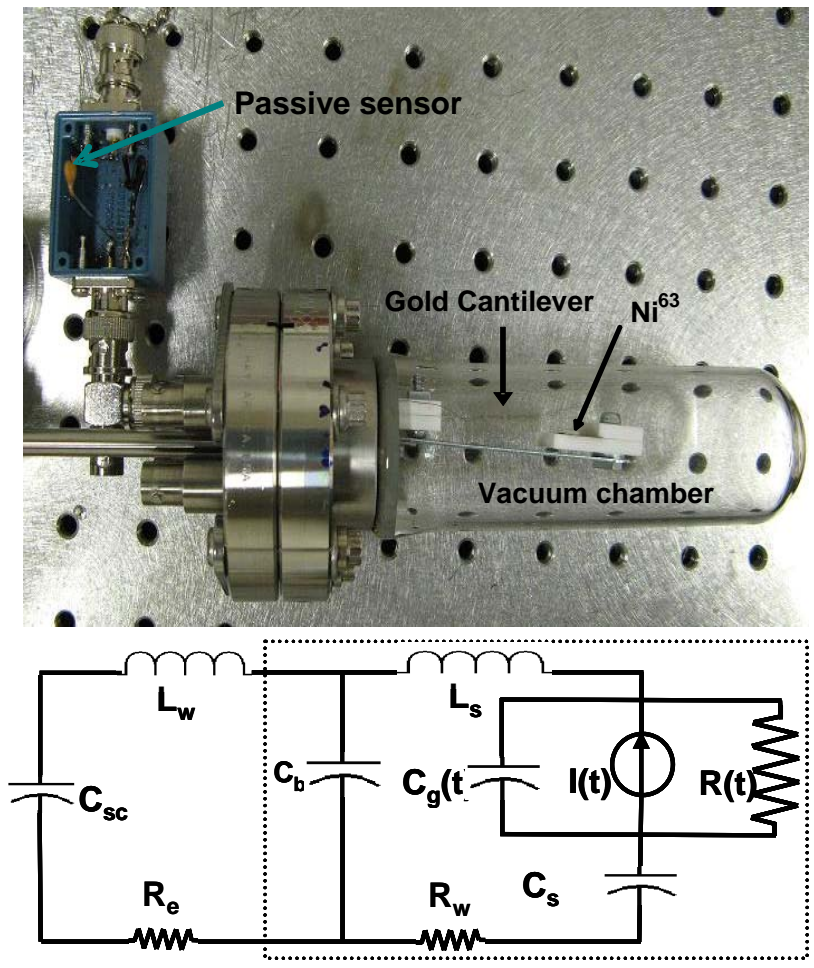

Figure 6. (a) Photograph, (b) equivalent LC circuit of the selfpowered wireless discharge system with external capacitance

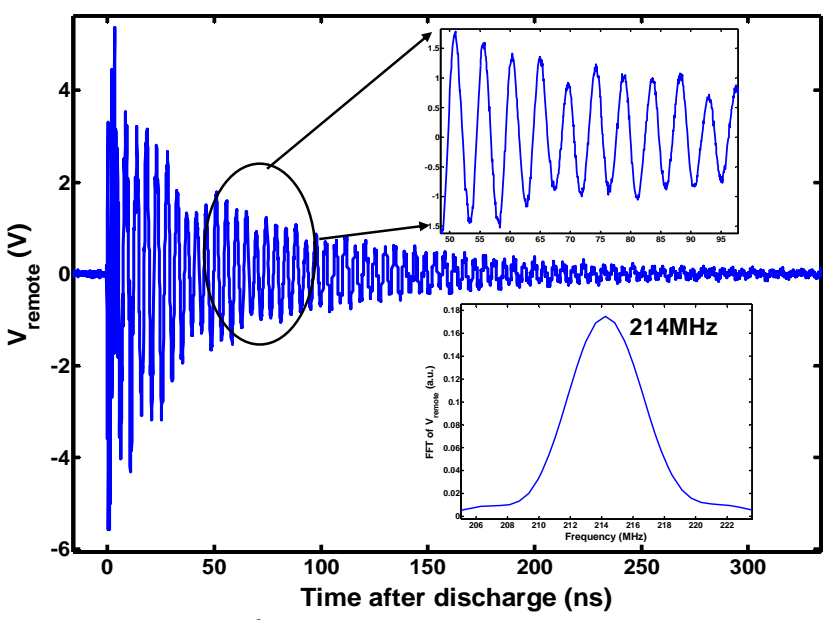

Figure 7. Magnitude and frequency of the remotely detected RF signal with $20 \mathrm{pF}$ external capacitance wireless RF sensor node

capacitance. $L_{w}$, and $R_{e}$, the inductance and resistance introduced by wire connections to the external capacitor, are measured to be $61 \mathrm{nH}$ and $0.8 \mathrm{Ohm}$ respectively using LCR meter (HP 4275A). To check the impact of the external capacitor, a $20 \mathrm{pF}$ capacitor is connected to $1.52 \mathrm{GHz}$ signal still exist for the first $10 \mathrm{~ns}$, an additional $214 \mathrm{MHz}$ the system, while the RF signal is measured remotely. While the RF signal is remotely detected upon cantilever discharge (Figure 7.) The RF signal lasts for more than 300 ns with a maximum of $500 \mathrm{~mW}$ detected $20 \mathrm{~cm}$ away from the chamber via the RF receiver. The SPICE simulation result of the new LC circuit (Figre 6(b)) with 20pF external capacitor agrees with the experimental measurement (Figure 8.)

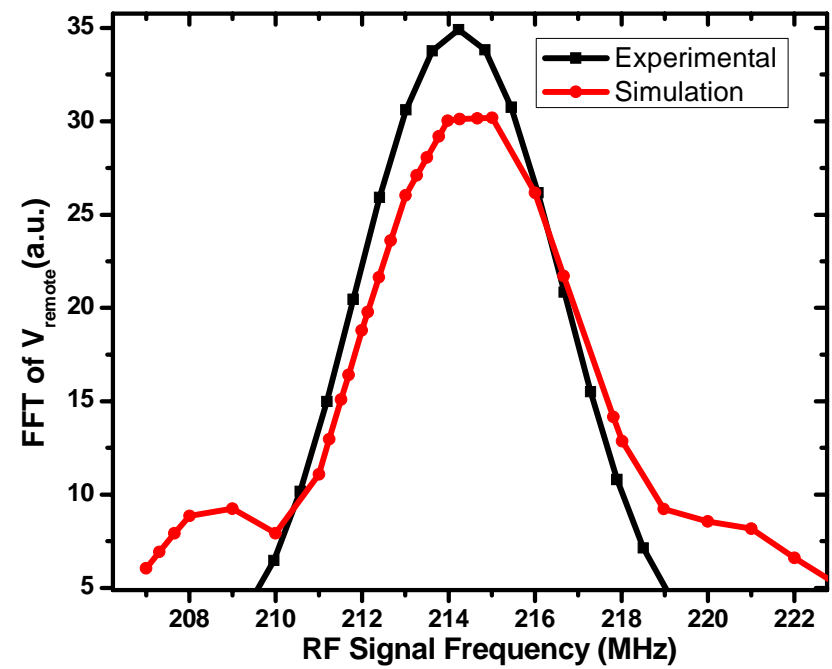

Figure 8. Resonance response of system with $20 \mathrm{pF}$ external capacitor from SPICE simulation and experimental measurement.

External capacitors with different values are then used to examine their impact on the frequency of the RF signal. The output $\mathrm{RF}$ signals are remotely detected with receiver and analyzed. The frequency of the RF signal is plotted versus the external capacitance in Figure 9. The frequency decreases with increasing external capacitance. When the capacitance increases from $12 \mathrm{pF}$ to $75 \mathrm{pF}$, a frequency shift of $63 \mathrm{MHz}$ is achieved $(239 \mathrm{MHz}$ to $176 \mathrm{MHz})$. The result of the SPICE simulation of LC circuit model matches the measurement results. Therefore, with the circuit model, the value of the external capacitor can be extracted from the 


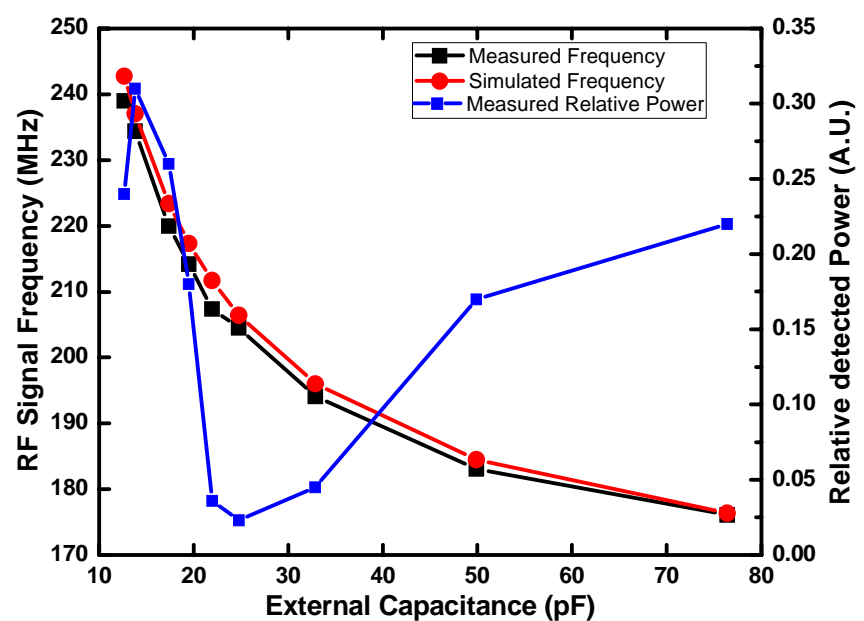

Figure 9. Experimental and simulated RF signal frequency with different external capacitance.

frequency of the remotely detected RF signal. The relative power of detected RF signals with different external capacitor values is also plotted in Figure 9. With cantilever reciprocation period almost stays the same, the transmitted RF signal power is consistent for all external capacitance values. However, the RF receiver has different gains at different frequencies, which causes the variation in detected RF signal power.

To implement a completely self-powered wireless sensor node, a capacitive polymer humidity sensor (Figure 10) is connected to the discharge system. The capacitance of the sensor at different humidity levels are first characterized (Figure 10). The different levels of relative humidity are generated by exposing the humidity sensor to different saturated salt solutions (with known relative humidity values) in a closed chamber. The capacitance of the sensor increases with the relative humidity. The RF signal is remotely measured at different humidity levels. The frequency of the signal is analyzed and plotted in Figure 11. A frequency shift of $4.27 \mathrm{MHz}$ is achieved when the humidity changes from $22 \%$ to 97\% (Figure 11). The experiment results match SPICE simulation outputs when the capacitance values of the humidity sensor are used in the LC circuit model in Figure 6(b).

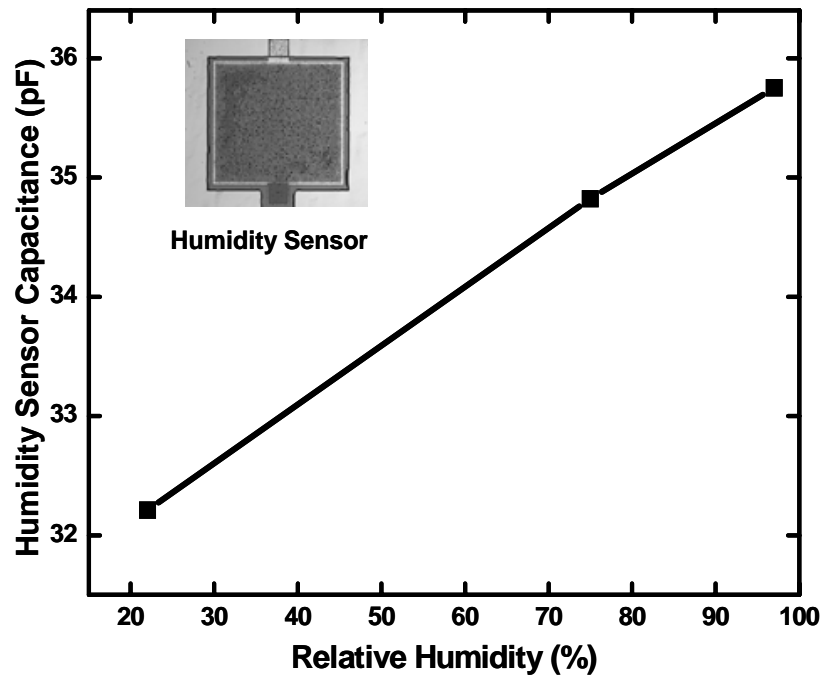

Figure 10. Measured humidity sensor capacitance at different relative humidity levels.

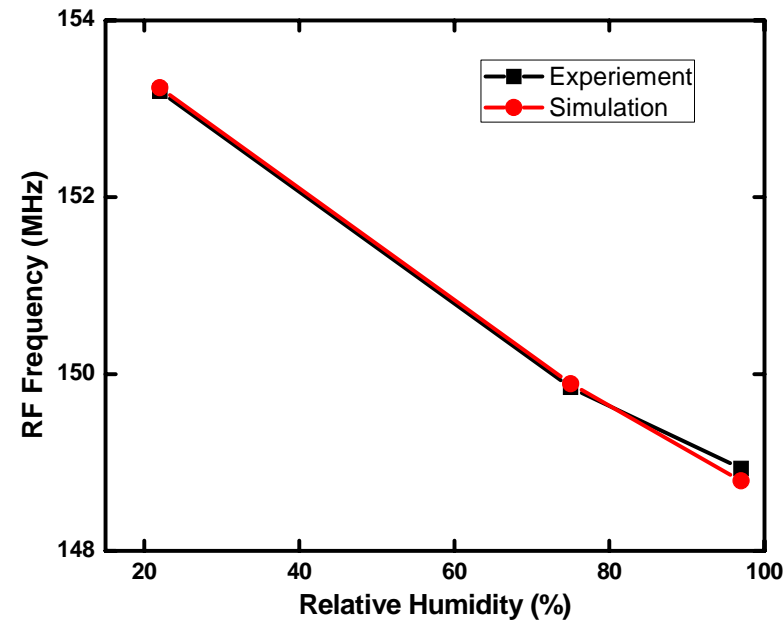

Figure 11. Measured remotely detected RF signal frequency at different relative humidity levels.

\section{CONCLUSIONS}

In this paper, we demonstrated a completely self-powered wireless sensor node which can transmit an RF signal coded with the environmental information gathered by a passive capacitive sensor. Sensor nodes that can operate for several decades and transmit data over RF-links at power of 100-1000 milliWatts without environmental energy harvesting can potentially revolutionize reliable long-term monitoring, such as water content in building foundations or detection of leached chemicals in chemical processing plants. These sensors need to be buried deep into structural constructs such as steel and concrete, where changing batteries or harvesting vibration or EM energy is not a reliable option. In the near future, efforts in developing microscale vacuum packages will be undertaken to reduce volume. Furthermore, transmission line equivalent models are also being developed to engineer desired frequency output from the all-selfpowered RF sensor node.

\section{ACKNOWLEDGEMENT}

This work was supported by DARPA-MTO, and contracted under the U. S. SPAWAR R7D center.

\section{REFERENCES}

[1] S. Roundy, D. Steingart, L. Frechette, P. Wright, and J. Rabaey, "Power Sources for Wireless Sensor Networks," Berlin, Germany, 2004, pp. 1-17

[2] R. Duggirala, S. Tin, and A. Lal, "3D Silicon Betavoltaics Microfabricated using a Self-Aligned Process for 5 Milliwatt/CC Average, 5 Year Lifetime Microbatteries,” Transducers 2007, Lyon, France, Jun. 2007, pp 279-282

[3] H. Li, A. Lal, J. Blanchard, and D. Henderson, "Self reciprocating Radioisotope powered Cantilever," Transducers, 2001, Munich, pp. 744-747

[4] R. Duggirala., A. Polcawich, E. Zakar, M. Dubey, and A. Lal, "MEMS Radioisotope-powered Piezoelectric Power Generator," MEMS 2006, Istanbul, Jan. 2006, pp.94-97.

[5] R. Duggirala, M. Kranz, C. Pollock, and A. Lal, "Selfpowered Humidity Sensor powered by Nickel-63 Radioisotope," 2006 Solid State Sensor and Actuator Workshop, Hilton Head Island, June 2007.

[6] S. Tin, R. Duggirala, R. Polcawich, M. Dubey, and A. Lal, "Self-powered Discharge-based Wireless Transmitter", MEMS 2008, Tucson, Jan. 2006, pp. 988 - 991 